\title{
Systemische Therapie des hepatozellulären Karzinoms
}

\author{
Martha M. Kirstein Arndt Vogel \\ Abteilung für Gastroenterologie, Hepatologie und Endokrinologie, Medizinische Hochschule Hannover, Deutschland
}

\author{
Schlüsselwörter \\ Hepatozeluläres Karzinom · Sorafenib · c-MET · Tivantinib
}

\section{Zusammenfassung}

Hintergrund: Das hepatozelluläre Karzinom (HCC) ist eine der häufigsten Tumorerkrankungen weltweit. Mit dem Multikinase-Inhibitor Sorafenib konnte erstmals ein signifikanter Überlebensvorteil bei Patienten mit fortgeschrittenem HCC gezeigt werden. Bis heute ist Sorafenib die einzige zugelassene systemisch wirksame Substanz für Patienten mit fortgeschrittenem HCC. Die weiteren Behandlungsoptionen für Patienten mit einem fortgeschrittenen HCC sind jedoch beschränkt. Es fehlen insbesondere Second-line-Therapien für Patienten, die Sorafenib nicht tolerieren und/oder hierunter progredient werden. Methode: Neue molekulare Therapien befinden sich in klinischer Entwicklung, bislang allerdings mit zumeist ernüchternden Ergebnissen. Ergebnisse: Untersuchungen der alternativen Angiogenese-Inhibitoren Sunitinib und Brivanib, der EGFR(epidermaler Wachstumsfaktor-Rezeptor)-Inhibitoren Erlotinib, Gefitinib, Lapatinib und Cetuximab sowie des MEK(Mitogen-aktivierte Proteinkinase)-Inhibitors Selumetinib waren bislang nicht zielführend. Erfolg versprechend erscheint die Inhibition von c-MET mit Tivantinib, die bei c-MET-überexprimierenden Patienten eine signifikante Verlängerung des Überlebens und der Zeit zur Progression erbrachte. Untersuchungen von anderen Zielgruppen wie unter anderem Inhibitoren von mTOR, PI3K/ Akt und IGFR befinden sich in teils noch früher klinischer Entwicklung und bleiben abzuwarten. Schlussfolgerungen: Die Entwicklung der systemischen Behandlung des HCC bleibt anspruchsvoll. Zunehmend zeichnet sich ab, dass bei HCC-Patienten eine schwierige Balance zwischen Toxizität der Behandlung und antitumoraler Wirkung besteht, die eine signifikante Verbesserung des Überlebens der Patienten ermöglicht. Eine Lösung dieses Dilemmas könnte in der Identifikation von prognostischen und prädiktiven Faktoren wie c-MET bestehen, die eine personalisierte und effektive Therapie in Subgruppen der HCCPatienten ermöglichen sollte.
Keywords

Hepatocellular carcinoma - Sorafenib - c-MET - Tivantinib

\section{Summary}

Systemic Therapy for Hepatocellular Carcinoma

Background: Hepatocellular carcinoma (HCC) is one of the most common and deadly malignancies worldwide. The multikinase inhibitor sorafenib has provided a survival benefit in patients with advanced HCC. Sorafenib remains the only approved agent for advanced HCC. There are only few systemic therapeutic options. No standardized second-line treatment exists for patients with progressive disease during sorafenib treatment or with intolerable side effects. Method: Novel molecular targets are under clinical development, with sobering outcomes so far. Results: Investigations of the alternative inhibitors of angiogenesis, i.e. sunitinib and brivanib, the EGFR (epidermal growth factor receptor) inhibitors erlotinib, gefitinib, lapatinib, and cetuximab as well as the MEK (mitogen-activated protein kinase) inhibitor selumetinib did not provide constructive results. Targeting c-MET with the associated inhibitor tivantinib appears to be a promising option. Overall survival and time to progression was significantly prolonged with tivantinib in patients overexpressing c-MET. Moreover, targets such as mTOR, PI3K/ Akt, and IGFR, among others, are under clinical development, though partially in early stages. The results remain to be seen. Conclusions: Systemic management of HCC remains challenging. Among HCC patients, a difficult balance between the toxicity of the treatment and the antitumoral effect can be ascertained, enabling a significant improvement of the survival of the patients. There is an urgent need for the identification of predictive and prognostic factors such as C-MET for a personalized and efficient treatment in subgroups of HCC patients.

\section{KARGER \\ Fax +497614520714 \\ Information@Karger.com}

www.karger.com (c) 2013 S. Karger GmbH, Freiburg

1662-6664/13/0292-0084\$38.00/0

Accessible online at:

www.karger.com/vim
Prof. Dr. med. Arndt Vogel

Abteilung für Gastroenterologie, Hepatologie und Endokrinologie

Medizinische Hochschule Hannover

Carl-Neuberg-Straße 1, 30625 Hannover, Deutschland

Vogel.Arndt@mh-hannover.de 


\section{Einleitung}

Das hepatozelluläre Karzinom (HCC) ist weltweit der siebthäufigste Tumor (am fünfthäufigsten bei Männern, am achthäufigsten bei Frauen) [1]. Aufgrund der immer noch unzureichenden Behandlungsoptionen für Patienten im fortgeschrittenen Tumorstadium ist das HCC die dritthäufigste tumorvermittelte Todesursache (www.iarc.fr). 2008 waren entsprechend der schlechten Prognose dieser Tumorerkrankung bei 748300 Neuerkrankungen 695900 Todesfälle zu verzeichnen.

Das HCC entsteht in 70-80\% aller Fälle auf dem Boden einer Leberzirrhose, die die Funktion der Leber in der Regel deutlich beeinträchtigt. Komplikationen der Leberzirrhose wie Blutungen aus Ösophagusvarizen, Aszites oder eine hepatische Enzephalopathie tragen zur hohen Mortalität und Morbidität der Patienten bei. Das über 5 Jahre kumulierte Risiko von zirrhotischen Patienten, ein HCC zu entwickeln, liegt zwischen 5 und 30\% [1]. Die hauptsächlichen Risikofaktoren für die Entstehung einer Leberzirrhose und damit eines HCC sind chronische Infektionen mit dem Hepatitis-B-Virus (HBV) oder Hepatitis-C-Virus (HCV) und Alkoholabusus. Zudem wird das HCC-Risiko durch nicht alkoholbedingte Leberverfettung (nonalcoholic fatty liver disease, NAFLD; nonalcoholic steatohepatitis, NASH) erhöht. Hinzu kommen seltenere Risikofaktoren wie Hämochromatose, Tyrosinämie, Autoimmunhepatitis, einige Porphyrien und Morbus Wilson. Diabetes, Adipositas, Rauchen, männliches Geschlecht und fortgeschrittenes Alter erhöhen zusätzlich das Risiko, an einem HCC zu erkranken.

Die «Barcelona Clinic Liver Cancer (BCLC)»-Klassifikation ist das gegenwärtig am häufigsten verwendete StagingSystem und gibt für jedes Stadium orientierende therapeuti- sche Empfehlungen [2-3]. Allerdings muss die individuelle Therapieempfehlung jedes HCC-Patienten in einem interdisziplinären Tumorboard getroffen werden. Die BCLC-Klassifikation unterscheidet zwischen fünf Stadien des HCC (BCLC-0 sowie BCLC-A bis BCLC-D) und bezieht dabei den Tumorstatus, die Leberfunktion und den allgemeinen Gesundheitszustand der Patienten ein. Grundsätzlich kann man nach therapeutischer Zielsetzung in ein Stadium mit kurativen (BCLC-0 und BCLC-A) und palliativen (BCLC-B bis BCLC-D) Optionen (Abb. 1) differenzieren.

Bis vor wenigen Jahren gab es bis auf bestmögliche unterstützende Maßnahmen (Best Supportive Care) keine palliative Behandlungsmöglichkeit für Patienten, die einer lokoregionalen Therapie nicht zugänglich waren. Herkömmliche Chemotherapien können zum einen durch die zumeist zugrunde liegende Leberzirrhose und die damit einhergehende Thrombozytopenie/Panzytopenie nicht eingesetzt werden. Zum anderen ist das HCC auch weitestgehend resistent gegen traditionelle Chemotherapien. Dies änderte sich mit der Verfügbarkeit des oralen Multikinase-Inhibitors Sorafenib, der sowohl die Tumorangiogenese als auch die Tumorproliferation und damit zwei charakteristische Stoffwechselwege des stark vaskularisierten HCC hemmt. Seine Angriffspunkte sind die Rezeptor-Tyrosinkinasen VEGFR 1-3, PDGF- $\beta$, Flt-3 und c-Kit, die am Anfang intrazellulärer Phosphorylierungskaskaden stehen und die Gefäßversorgung des Tumors vermitteln. Darüber hinaus inhibiert Sorafenib das Raf-Protein, ein Schlüsselmolekül des Mitogen-aktivierten Proteinkinase-Signalwegs (MAPK), der eine zentrale Rolle bei der Zellproliferation spielt. Für die europäischen Fachgesellschaften repräsentiert Sorafenib in ihren aktuellen Richtlinien die einzige HCC-Therapie von gleichzeitig höchstem Evidenzlevel und Empfehlungsgrad. Nichtsdestotrotz ist der klinische
Abb. 1. Einteilung der Stadien und Behandlung nach der BCLC-Klassifikation (PST = Performance-Status, RF/PEI = Radiofrequenzablation/perkutane Ethanolinjektion, TACE = transarterielle Chemoembolisation) (aus [38], mit freundlicher Genehmigung des Georg Thieme Verlags, Stuttgart; modifiziert nach [2]).

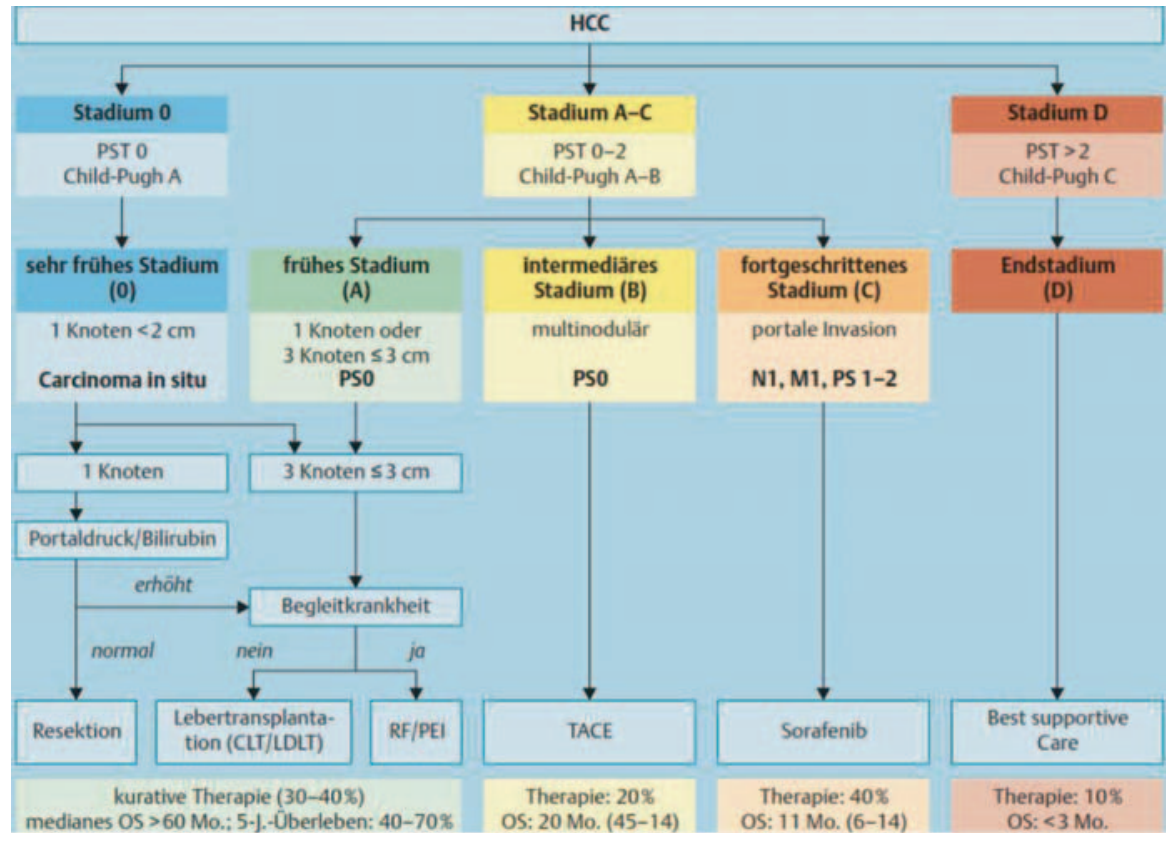




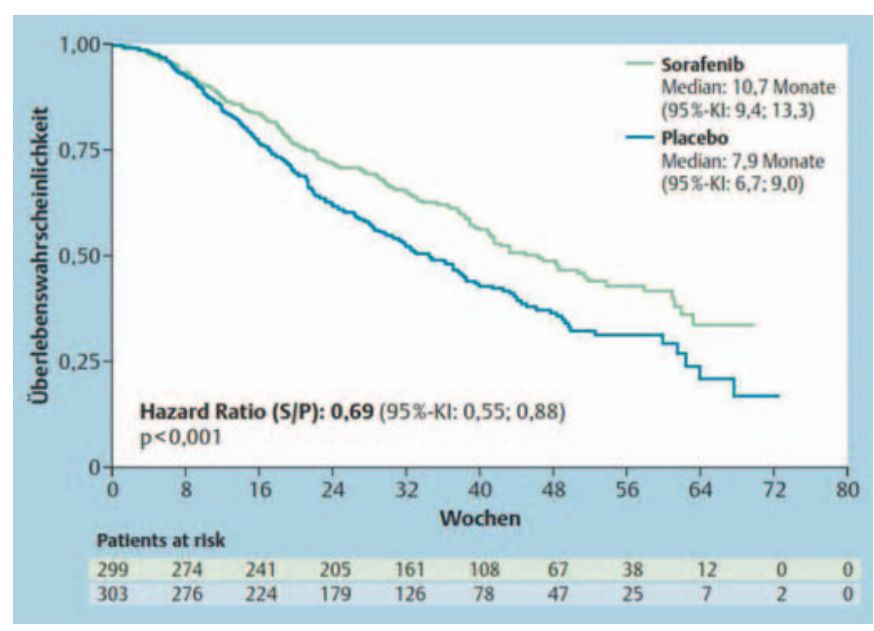

Abb. 2. Sorafenib bei Patienten mit fortgeschrittenem HCC: Gesamtüberleben (aus [38], mit freundlicher Genehmigung des Georg Thieme Verlags, Stuttgart; modifiziert nach [5]).

Benefit von Sorafenib leider nur begrenzt, und die Behandlungsoptionen des fortgeschrittenen HCC müssen dringend verbessert und erweitert werden.

Die Zulassung von Sorafenib führte zu einem starken Anstoß der Arzneimittelentwicklung im HCC-Bereich. Eine große Anzahl verschiedener molekularer Substanzen, die in bekannte Signalwege der Hepatokarzinogenese eingreifen oder auch gegen neue Zielmoleküle gerichtet sind, befinden sich derzeit in unterschiedlichen Phasen der klinischen Entwicklung. Hierbei lassen sich prinzipiell drei Vorgehensweisen unterscheiden:

- Die Kombination von Sorafenib mit bekannten, antiproliferativ und/oder antiangiogenetisch wirksamen Arzneimitteln.

- Die Testung von alternativen, antiangiogenetisch wirksamen Arzneimitteln.

- Die Identifikation und Validierung von alternativen molekularen Zielen, die wichtige andere Tumor-Signalwege beeinflussen.

Im Folgenden werden wir die Ergebnisse publizierter Studien von Sorafenib bis hin zu aktuellen Untersuchungen von systemischen Therapieoptionen im fortgeschrittenen HCC zusammenfassen.

\section{Ergebnisse}

\section{Sorafenib}

Auf der Basis einer Phase-II-Studie [4], die bei 137 Patienten mit fortgeschrittenem HCC eine lebensverlängernde Wirkung von Sorafenib gezeigt hatte, wurden die Wirksamkeit und die Verträglichkeit des Präparats in der multizentrischen, randomisierten, doppelblinden und placebokontrollierten SHARP-Studie (Sorafenib HCC Assessment Randomized
Protocol Trial) geprüft [5]. Diese Phase-III-Studie schloss 602 Patienten in 121 Behandlungszentren aus 21 Ländern (Europäische Union/EU, Nord- und Südamerika, Australien) mit überwiegend fortgeschrittenem HCC ein (18\% der Sorafenibund $17 \%$ der Placebo-Gruppe befanden sich erst im intermediären Stadium BCLC-B). Weitere Einschlusskriterien waren eine gut erhaltene Leberfunktion (Child-Pugh A), ein ECOGPerformance-Status von höchstens 2 und eine Lebenserwartung von mindestens 12 Wochen. 299 Patienten wurden in den Sorafenib-Arm (400 mg peroral 2-mal täglich) und 303 Patienten in den Placebo-Arm der Studie randomisiert.

Bereits nach der zweiten Interimsanalyse wurde die Studie abgebrochen, weil sich signifikante Überlebensvorteile in der Sorafenib-Gruppe zeigten (Abb. 2). Die mediane Gesamtüberlebenszeit unter Sorafenib betrug 10,7 Monate gegenüber 7,9 Monaten in der Placebo-Gruppe (Hazard Ratio (HR) 0,$69 ; 95 \%$-Konfidenzintervall (KI) $0,55-0,87 ; \mathrm{p}=0,00058$ ). Sorafenib verbesserte auch die mittlere Zeit bis zur radiologisch ermittelten Tumorprogression von 2,8 auf 5,5 Monate (HR $0,58 ; 95 \%$-KI $0,44-0,74 ; p=0,000007)$. Kein statistisch signifikanter Unterschied zwischen beiden Gruppen ergab sich hinsichtlich der ermittelten Zeit bis zur symptomatischen Progression (4,1 vs. 4,9 Monate; HR 1,08; $95 \%$-KI 0,88-1,31; $\mathrm{p}=$ 0,77). Sorafenib zeigte in allen Subgruppen einen Vorteil im Gesamtüberleben gegenüber Placebo [6-9].

Die Ergebnisse der SHARP-Studie veranlassten die europäische und die amerikanische Arzneimittelbehörde, Sorafenib Ende 2007 als erstes Medikament zur Behandlung des HCC (EU) bzw. des nicht resezierbaren HCC (USA) zuzulassen.

In der SHARP-Studie wurde eine Behandlungsunterbrechung aufgrund von Nebenwirkungen bei $38 \%$ der mit Sorafenib behandelten Patienten gegenüber $37 \%$ der Patienten in der Kontrollgruppe notwendig. Die meisten Nebenwirkungen waren dermatologischer, gastrointestinaler und konstitutioneller Art, wobei die häufigsten Toxizitäten vom Grad 3 oder 4 Diarrhö (Sorafenib 8\%, Placebo 2\%) und Hand-Fuß-Hautreaktionen (Sorafenib $8 \%$, Placebo $<1 \%$ ) waren. Im klinischen Alltag vermindern insbesondere die Hand-Fuß-Hautreaktionen die Lebensqualität deutlich und führen häufig zu Unterdosierungen oder Therapieunterbrechungen (Abb. 3). Interessanterweise konnte in einer großen randomisierten Studie der prophylaktische Effekt harnstoffhaltiger Salben gegenüber Best Supportive Care bei Patienten mit Hand-FußHautreaktionen unter der Behandlung mit Sorafenib bei fortgeschrittenem HCC gezeigt werden, sodass analog zur präventiven Behandlung von Patienten mit Kolonkarzinom unter EGFR(epidermaler Wachstumsfaktor-Rezeptor)-Antikörpertherapie diese auch bei HCC-Patienten erfolgen kann [10].

Für differenziertere Aufschlüsse über die HCC-Therapie mit Sorafenib im klinischen Alltag wurden im Rahmen der GIDEON-Studie (Global Investigation of Therapeutic Decisions in Hepatocellular Carcinoma and of its Treatment with Sorafenib) 2770 Patienten aus 37 Ländern (37\% EU) zwi- 
Abb. 3. Der Preis der Therapie: Das HandFuß-Syndrom unter der Behandlung mit Sorafenib (aus [39]; Copyright S. Karger AG, Basel).
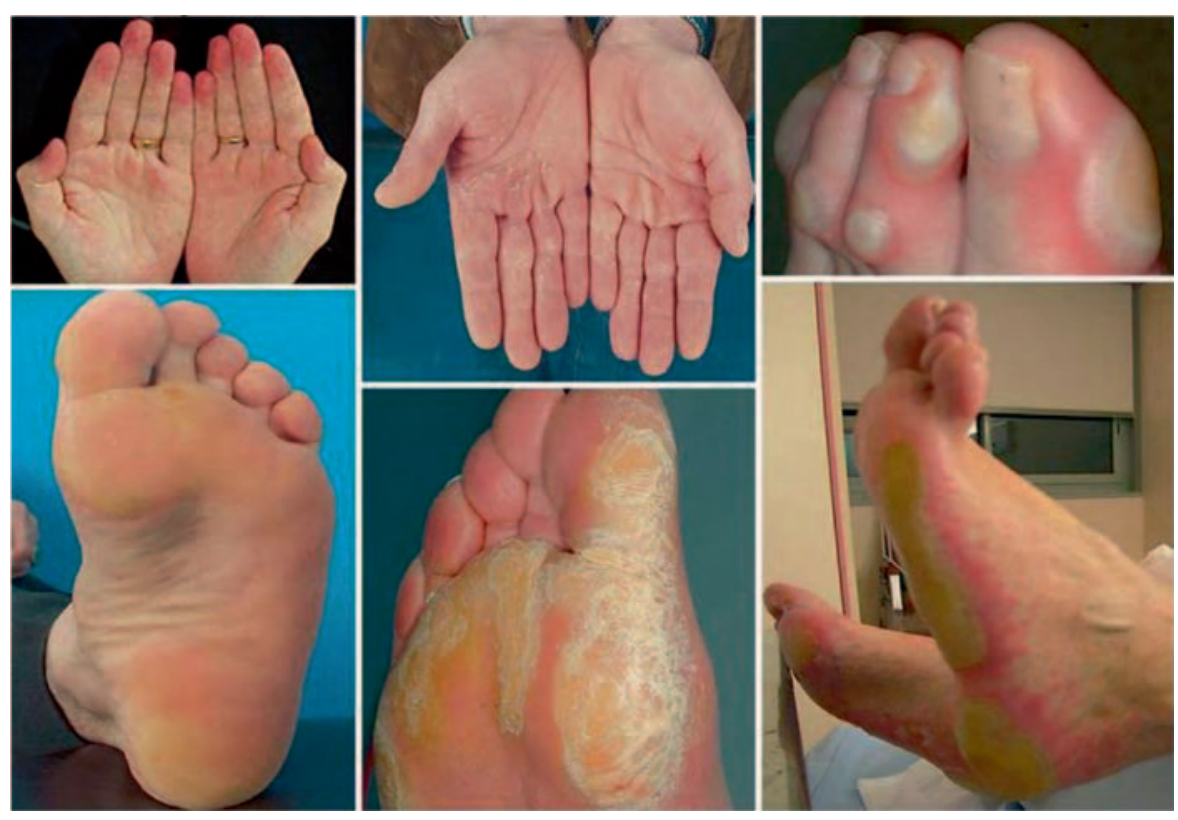

schen Januar 2009 und Dezember 2010 für eine systemische Therapie mit Sorafenib ausgewählt [11]. Bislang hat sich der in der SHARP-Studie gezeigte Überlebensvorteil und die Verzögerung zur Progression unter Sorafenib bestätigt - und dies insbesondere für Patienten mit gut erhaltener Leberfunktion (Child-Pugh A). Weiterhin ist unklar, ob die Therapie mit Sorafenib auch zu einem Überlebensvorteil bei Patienten im Stadium Child-Pugh B führt. Entsprechend wird in der aktuellen S3-Leitlinie die Empfehlung zur Therapie mit Sorafenib primär für Patienten mit Child-Pugh A ausgesprochen. Bei Patienten im frühen Stadium von Child-Pugh B (7 Punkte) muss eine individuelle Therapieentscheidung getroffen werden.

\section{Sunitinib}

Nach der erfolgreichen Phase-III-Studie mit Sorafenib wurde eine Reihe weiterer antiangiogenetisch wirksamer Substanzen für die Therapie des HCC sowohl bei therapienaiven Patienten als auch bei Patienten nach Sorafenib-Versagen oder -Unverträglichkeit untersucht (Tab. 1).

Erste Erfolg versprechende Ergebnisse aus Phase-II-Studien lieferten die Grundlage für eine Phase-III-Vergleichsstudie mit dem antiangiogenetisch und antiproliferativ wirksamen Multikinase-Inhibitor Sunitinib (SUN1170-HCCStudie) [12, 13], in deren beiden Arme 1073 Patienten - im Gegensatz zur SHARP-Studie auch aus dem asiatischen Raum stammend - randomisiert aufgenommen wurden (544 mit Sorafenib, 529 mit Sunitinib; 24\% nichtasiatische Patienten). Das primäre Ziel der Studie war es, die Überlegenheit oder Nichtunterlegenheit von Sunitinib bezüglich der Gesamtüberlebenszeit gegenüber Sorafenib nachzuweisen. Dieses Ziel wurde jedoch nicht erreicht, da die Studie 2010 vorzeitig abgebrochen wurde, nachdem das unabhängige DatenMonitoring-Komitee (DMC) eine ungünstige Risiko-Nutzen-
Tab. 1. Alternative, getestete antiangiogenetische Inhibitoren im $\mathrm{HCC}$ (nach [41-43])

\begin{tabular}{llllll}
\hline & VEGFR & PDGFR & FGFR & VEGF & c-KIT \\
\hline Sorafenib & 2,3 & $\checkmark$ & & & $\checkmark$ \\
Sunitinib & 1,2 & $\checkmark$ & & $\checkmark$ \\
Axitinib & $1,2,3$ & & & \\
Brivanib & $1,2,3$ & & $1,2,3$ & \\
Linifanib & $1,2,3$ & & & \\
BIBF 1120 & $1,2,3$ & $\checkmark$ & $1,2,3$ & \\
Bevacizumab & & & & $\checkmark$ & \\
Ramucirumab & 2 & & & & \\
\hline
\end{tabular}

Bewertung für die Sunitinib-Gruppe vorgenommen hatte. In dieser waren die Frequenz und der Schweregrad der Nebenwirkungen so hoch, dass dort bei einem vorher geplanten Sicherheitsreview $18 \%$ behandlungsbedingte Todesfälle festgestellt wurden. Die anschließende Analyse ergab ein medianes Gesamtüberleben von 7,9 Monaten in der Sunitinib- und von 10,2 Monaten in der Sorafenib-Gruppe (HR 1,30; 95\%-KI $1,13-1,50 ; \mathrm{p}=0,0010)$ und bestätigte somit die nur auf der SHARP-Studie basierenden Zulassungsdaten für Sorafenib.

In der Subgruppe der nichtasiatischen Patienten mit chronischer Hepatitis $\mathrm{C}$ bestätigte sich interessanterweise die besondere Wirksamkeit von Sorafenib in dieser Patientengruppe - mit einer mittleren Überlebenszeit in der SorafenibGruppe von sogar 18,3 Monaten gegenüber 8,6 Monaten in der Sunitinib-Gruppe (HR 1,76; 95\%-KI 0,99-3,10; p = 0,0544) (Abb. 4).

\section{Brivanib}

Eine weitere interessante antiangiogenetisch wirksame Substanz, die sich derzeit in klinischer Testung befindet, ist Brivanib. Brivanib ist ein oral verfügbarer, selektiver, dualer 
Abb. 4. SHARP-Subgruppenanalyse: Gesamtüberleben $(\mathrm{KI}=$ Konfidenzintervall, $\mathrm{TACE}=$ transarterielle Chemoembolisation, $\mathrm{HCV}=$ Hepatitis-C-Virus, MVI = mikrovaskuläre Invasion, EHS = extrahepatische Ausweitung) (aus [38], mit freundlicher Genehmigung des Georg Thieme Verlags, Stuttgart; modifiziert nach $[6-9,40])$

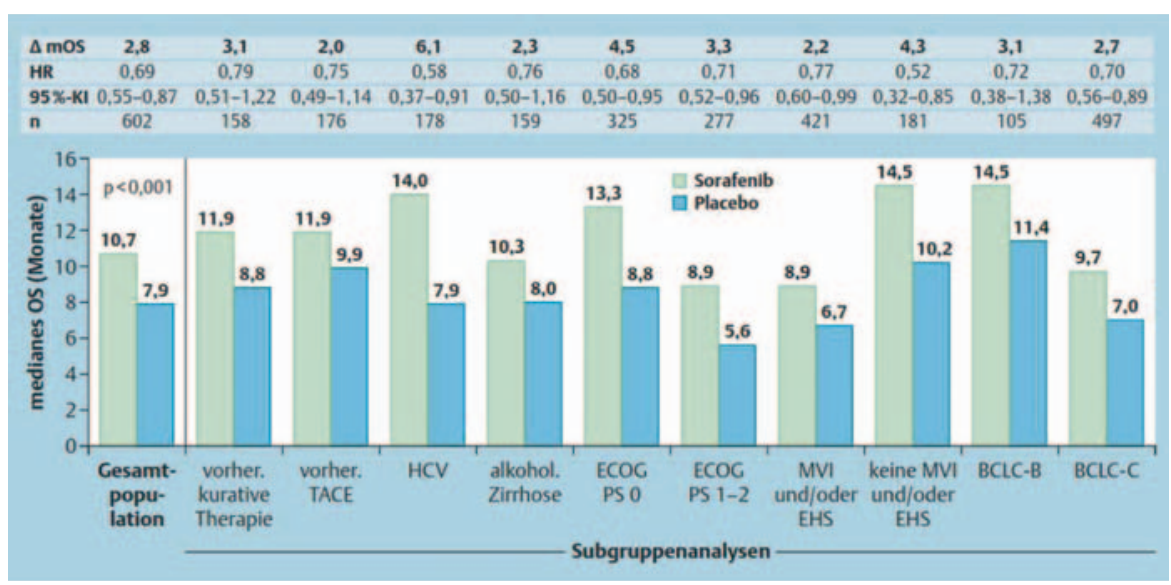

Inhibitor der VEGF(vaskulärer endothelialer Wachstumsfaktor)- und FGF(Fibroblasten-Wachstumsfaktor)-Rezeptoren, der das Tumorwachstum direkt und indirekt beeinflusst [1417]. In präklinischen Studien konnten die antitumorösen Eigenschaften von Brivanib in multiplen Tumoren einschließlich des HCC in Sorafenib-resistenten Tumormodellen gezeigt werden [14, 18, 19]. Diese präklinischen Ergebnisse wurden auf die Induktion von FGF bei Sorafenib-resistenten Patienten zurückgeführt und lieferten die Grundlage für eine offene Phase-II-Studie, in der Brivanib (800 mg täglich) zum einen als Second-line-Therapie nach gescheiterter antiangiogenetischer Behandlung [20] und zum anderen als First-lineTherapie bei Patienten mit fortgeschrittenem HCC getestet wurde [21]. Als primäre Endpunkte wurden unter anderem das progressionsfreie Überleben und das Gesamtüberleben vereinbart. In beiden Kohorten konnte bei 40-58\% zumindest eine Stabilisierung (stable disease) der Erkrankung erreicht werden. Das mediane Gesamtüberleben war 9,8 Monate $(95 \%-K I$ 5,5-13,2) in der Second-line- bzw. 10 Monate $(95 \%$ KI 6,8-15,2) in der First-line-Kohorte. Die häufigsten Nebenwirkungen waren Fatigue, Appetitlosigkeit, Übelkeit, Diarrhö und Hypertension. Insgesamt zeigte Brivanib ein günstiges Sicherheitsprofil.

Basierend auf diesen Ergebnissen wurden zwei große Phase-III-Erstlinien- und -Zweitlinien-Studien beim HCC initiiert. In der doppelblinden, multizentrischen BRISK-PS-Studie wurde die Wirksamkeit von Brivanib gegenüber Best Supportive Care bei Patienten mit fortgeschrittenem HCC nach Versagen von oder Intoleranz gegenüber Sorafenib untersucht (ClinicalTrials.gov-Identifier: NCT00825955). In dieser Studie wurden 395 Patienten aus Europa (42\%), Asien (41\%) und Amerika (17\%) eingeschlossen. Die große Mehrheit (85\%) bestand aus Männern mit einem medianen Alter knapp über 60 Jahren. Stratifiziert wurde nach Intoleranz gegenüber oder Progress unter Sorafenib, nach ECOG-Performance-Status 0 versus 1-2 und dem Vorhandensein oder Fehlen von makroskopischer Gefäßinvasion und/oder extrahepatischen Metastasen. Ende 2011 zeigte sich allerdings, dass das Gesamtüberleben unter Brivanib im Vergleich zu Placebo nicht signifikant verbessert werden konnte. Überraschenderweise hatten insbesondere Patienten mit großem Tumor (10 $\mathrm{cm}$ ), hohem Alpha-Fetoprotein, extrahepatischer Manifestation und/oder makroskopischer Gefäßinvasion nicht von der Behandlung mit Brivanib profitiert.

In der zweiten Phase-III-Studie wurde Brivanib gegenüber Sorafenib als First-line-Behandlung bei Patienten mit fortgeschrittenem HCC (BRISK-FL-Studie) untersucht (ClinicalTrials.gov-Identifier: NCT00858871). 1050 Patienten mit fortgeschrittenem HCC ohne vorherige systemische Behandlung wurden in der randomisierten, doppelblinden, multizentrischen Phase-III-Studie eingeschlossen. Allerdings wurde auch hier der primäre Endpunkt, also eine Nichtunterlegenheit von Brivanib gegenüber Sorafenib, bei diesem gut ausgewogenen Patientenkollektiv nicht erreicht. Basierend auf diesen sehr enttäuschenden Daten wurden alle Studienprogramme von Brivanib bei HCC eingestellt.

\section{EGFR-Signalweg}

Neben den antiangiogenetischen Therapien wurden zunehmend auch andere molekulare Signalwege als mögliche Angriffspunkte für eine systemische Therapie bei Patienten mit fortgeschrittenem HCC untersucht.

In Anbetracht der wichtigen Rolle des EGFR-Signalwegs bei vielen Tumorentitäten und den dort erfolgreich beendeten Studien stellten die entsprechenden molekularen Inhibitoren hierbei eine der als Erstes untersuchten Gruppen dar. Alle bislang publizierten Phase-II- und -III-Studien, die die EGFR-Inhibitoren Erlotinib [22-24], Gefitinib [25], Lapatinib [26] und Cetuximab [27] bei Patienten mit fortgeschrittenem HCC untersuchten, haben bislang allerdings keine überzeugende antitumorale Wirksamkeit nachweisen können.

Erlotinib ist ein selektiver Inhibitor der Tyrosinkinase-Domäne des EGFR, der beim fortgeschrittenen oder metastasierten nichtkleinzelligen Bronchialkarzinom (non-small-cell lung carcinoma, NSCLC) zugelassen ist und auch bereits beim HCC in kleineren Phase-II-Studien getestet wurde [22-24]. Die Ergebnisse dieser Studien ließen vermuten, dass diese Substanz in der Kombination mit Sorafenib eine Aktivität 
beim HCC hat, und veranlassten die Initiierung der SEARCH-Studie, einer internationalen, multizentrischen, placebokontrollierten Phase-III-Studie, in der 720 Patienten mit fortgeschrittenem HCC randomisiert wurden. Verglichen wurde primär das mittlere Gesamtüberleben unter Sorafenib plus Placebo gegenüber Sorafenib plus Erlotinib. Im Juli 2012 wurde bekannt, dass die Kombination mit Erlotinib kein verbessertes Überleben im Vergleich zu einer alleinigen Therapie mit Sorafenib erbrachte, sodass insgesamt nicht zu erwarten ist, dass Inhibitoren des EGFR-Signalwegs eine Rolle bei der Therapie des HCC spielen werden.

\section{RAF/MEK/ERK-Signalweg}

Die molekularen Mechanismen der Wirksamkeit von Sorafenib sind bis heute nicht gut verstanden. Aufgrund seiner Aktivität gegenüber dem RAF-Signalweg, der in 50-60\% der HCCs aktiviert ist, entwickelte sich in den vergangenen Jahren ein wachsendes Interesse an diesem RAF/MEK(Mitogenaktivierte Proteinkinase)/ERK-Signalweg, und der oral verfügbare Inhibitor der MEK-Tyrosinkinase Selumetinib wurde in einer Phase-II-Studie bei Patienten mit fortgeschrittenem HCC ohne vorherige Behandlung getestet [28]. Entgegen der großen Erwartungen konnte in dieser Studie allerdings kein radiologisches Ansprechen verzeichnet und die Zeit zur Progression nicht verlängert werden.

\section{mTOR-Signalweg}

«Mammalian target of rapamycin» (mTOR) ist ein zentraler Regulator einer Reihe von zellulären Prozessen, die von hohem onkologischen Interesse sind. In vielen Tumorentitäten, inklusive dem HCC, wurde zudem eine deutliche Aktivierung dieses Signalwegs nachgewiesen und korreliert beim HCC z.B. mit dem Risiko eines Rezidivs nach Resektion [29]. Präklinische Studien haben zudem gezeigt, dass mTOR-Inhibitoren effektiv das Zellwachstum und die Gefäßinvasion in HCC-Zelllinien in vitro und in HCC-Tumormodellen in vivo hemmen [29-31]. Verschiedene mTOR-Inhibitoren (Sirolimus, Temsirolimus und Everolimus) sind derzeit in klinischer Erprobung beim HCC. In der SilVER05-Studie wird untersucht, ob durch Sirolimus im Vergleich zu einer mTORInhibitor-freien Immunsuppression das Risiko eines HCCRezidivs nach Lebertransplantation verringert werden kann. In diese Studie konnten alle geplanten Patienten eingeschlossen werden; diese befinden sich derzeit in der Nachbeobachtungsphase (Sirolimus-containing versus mTOR-inhibitorfree immunosuppression in patients undergoing liver transplantation for hepatocellular carcinoma; ClinicalTrials.govIdentifier: NCT00355862). In der palliativen Situation wurde in einer großen Phase-III-Studie die Wirksamkeit von Everolimus bei Patienten mit Sorafenib-Versagen/Unverträglichkeit bei über 700 Patienten untersucht (Evolve-1-Studie, ClinicalTrials.gov-Identifier: NCT00345839). Diese Studie wurde nicht vorzeitig beendet; die Ergebnisse werden für 2013 erwartet.
Tab. 2. Ergebnisse der randomisierten Phase-II-Studie mit Tivantinib (nach [37])

\begin{tabular}{|c|c|c|c|c|}
\hline & Tivantinib & Placebo & $\operatorname{HR}\left(\mathrm{KI}^{\mathrm{a}}\right)$ & p-Wert \\
\hline \multicolumn{5}{|c|}{ Median TTP, Wochen } \\
\hline ITT & 6,9 & 6,0 & $0,64(0,43-0,94)$ & 0,04 \\
\hline MET $^{\text {hoch }}$ & 11,7 & 6,1 & $0,43(0,19-0,97)$ & 0,03 \\
\hline \multicolumn{5}{|c|}{ Median OS, Monate } \\
\hline ITT & 6,6 & 6,2 & $0,90(0,57-1,40)$ & 0,63 \\
\hline MET $^{\text {hoch }}$ & 7,2 & 3,8 & $0,38(0,18-0,81)$ & 0,01 \\
\hline
\end{tabular}

${ }^{\text {a } 90 \%-K I ~ f u ̈ r ~ p r i m a ̈ r e ~ T T P ; ~ 95 \%-K I ~ f u ̈ r ~ a n d e r e ~ E n d p u n k t e . ~}$

TTP = Mittlere Tumorprogressionszeit; ITT = Intent-to-TreatPopulation; OS = Gesamtüberleben.

\section{C-MET/HGF-Signalweg}

Eine Substanzgruppe von derzeit sehr hohem Interesse bei einer Vielzahl von Tumorentitäten sind Inhibitoren der Rezeptor-Tyrosinkinase c-MET mit seinem Liganden, dem Hepatozyten-Wachstumsfaktor (hepatocyte growth factor, HGF). C-MET spielt eine wichtige Rolle im Zell- und Tumorwachstum, und eine Überexpression/Aktivierung von c-MET konnte beim HCC in einer Reihe von retrospektiven Analysen gezeigt werden [32-35]. Cabozantinib (XL184) ist ein oral verfügbarer, dualer MET/VEGFR2-Inhibitor. Dieser wurde gegenüber Placebo in einer Phase-II-Diskontinuitätsstudie bei 41 Child-Pugh-A-Patienten mit progredientem HCC und maximal einer einzigen Vorbehandlung entweder als Firstoder Second-line-Behandlung untersucht [36]. Als primäre Endpunkte wurden die Gesamt-Ansprechrate nach mRECIST und das progressionsfreie Überleben festgelegt. Mit einem progressionsfreien Überleben von 4,4 Monaten und einem medianen Gesamtüberleben von 15,1 Monaten zeigte Cabozantinib eine beachtliche antitumorale Wirkung bei Patienten mit fortgeschrittenem HCC.

Ein weiterer Erfolg versprechender c-MET-Inhibitor ist Tivantinib (ARQ197), ein selektiver, non-ATP-kompetitiver Inhibitor der MET-Tyrosinkinase. Tivantinib wurde kürzlich in einer multizentrischen, placebokontrollierten, doppelblinden Phase-II-Studie untersucht [37]. 107 Patienten mit fortgeschrittenem HCC und Child-Pugh-A-Zirrhose, die unter First-line-Therapie progredient oder dieser gegenüber intolerant waren, wurden in die Studie eingeschlossen. Primärer Endpunkt dieser Studie war die Zeit bis zur radiologisch gesicherten Progression. Erfreulicherweise wurde schon vorab festgelegt, Tumorgewebe zentral zu asservieren und zwecks Beurteilung der c-MET-Expression immunhistochemisch aufzuarbeiten. In der gesamten Population konnte keine Verbesserung des Überlebens durch die Therapie mit Tivantinib erreicht werden. Interessanterweise konnte in dieser Studie allerdings c-MET prospektiv als negativer prognostischer Faktor identifiziert werden; d.h., Patienten mit einer Überexpression von c-MET wiesen ein signifikant kürzeres Überleben auf als jene ohne C-MET-Expression. Durch die Thera- 
pie mit Tivantinib bei c-MET-überexprimierenden Patienten konnte eine signifikante Verlängerung des Gesamtüberlebens von 7,2 Monaten im Vergleich zu 3,8 Monaten in der PlaceboGruppe sowie eine signifikante Verlängerung der Zeit zur Progression erreicht werden (Tab. 2). Basierend auf diesen positiven Daten wird in Kürze eine Phase-III-Studie zur Evaluierung von Tivantinib als Second-line-Behandlung bei c-MET-überexprimierenden Patienten begonnen.

\section{Schlussfolgerungen}

Das HCC ist eine der häufigsten Tumorerkrankungen und tumorvermittelten Todesursachen weltweit. Mit dem Multikinase-Inhibitor Sorafenib konnte erstmals ein signifikanter Überlebensvorteil bei Patienten mit fortgeschrittenem HCC gezeigt werden. Bis heute ist Sorafenib die einzige zugelassene systemisch wirksame Substanz für Patienten mit fortgeschrittenem HCC. Die Behandlungsoptionen sind jedoch beschränkt. Es fehlen Second-line-Therapien für Patienten, die Sorafenib nicht tolerieren und/oder hierunter progredient werden.

Neue molekulare Therapien befinden sich in klinischer Entwicklung, bislang allerdings mit zumeist negativen Ergeb- nissen. Die getesteten alternativen Angiogenese-Inhibitoren Sunitinib und Brivanib konnten in Phase-III-Studien keine Verlängerung des Gesamtüberlebens zeigen. Eine andere, initial vielversprechende Zielgruppe stellten die EGFR-Inhibitoren dar. Leider erwiesen sich auch diese weder als Alternative zu noch als Option zur Kombination mit Sorafenib. Andere interessante molekulare Substanzen wie Inhibitoren von mTOR, PI3K/Akt und IGFR befinden sich in teils noch früher klinischer Entwicklung.

Aktuell Erfolg versprechend erscheint die Inhibition von c-MET. Eine Phase-II-Studie mit dem c-MET-Inhibitor Tivantinib zeigte erstmals bei c-MET-überexprimierenden Patienten eine signifikante Verlängerung des Überlebens sowie der Zeit zur Progression. Die Entwicklung der systemischen Behandlung des HCC bleibt anspruchsvoll. Zunehmend wird jedoch ersichtlich, dass die große Herausforderung in der Identifikation von prognostischen und prädiktiven Faktoren für eine personalisierte Therapie besteht.

\section{Disclosure Statement}

Die Verfasser haben keinen Interessenkonflikt zu offenbaren.

\section{Literatur}

1 El-Serag HB: Hepatocellular carcinoma. N Engl J Med 2011;365:1118-1127.

2 European Association for the Study of the Liver; European Organisation for Research and Treatment of Cancer: EASL-EORTC clinical practical guidelines: management of hepatocellular carcinoma. J Hepatol 2012;56:908-943.

3 Bruix J, Sherman M: Management of hepatocellular carcinoma: an update. Hepatology 2011;53: 1020-1022.

4 Abou-Alfa GK, Schwartz L, Ricci S, Amadori D, Santoro A, Figer A, De Greve J, Douillard JY, Lathia C, Schwartz B, Taylor I, Moscovici M, Saltz LB: Phase II study of sorafenib in patients with advanced hepatocellular carcinoma. J Clin Oncol 2006;24:4293-4300.

5 Llovet JM, Ricci S, Mazzaferro V, et al.; SHARP Investigators Study Group: Sorafenib in advanced hepatocellular carcinoma. N Engl J Med 2008;359: 378-390.

6 Galle P, Blanc J, Van Laethem J-L, Marrero J, Beaugrand M, Moscovici M, Shan M, Nadel A, Voliotis D, Bruix J, Lovet JM: Efficacy and safety of sorafenib in patients with advanced hepatocellular carcinoma and prior anti-tumor therapy: a subanalysis from the sharp trial. J Hepatol 2008;48 (suppl 2):S372.

7 Bolondi L, Caspary W, Bennouna J, Thomson B, Van Steenbergen W, Degos F, Shan M, Moscovici M, Llovet J, Bruix J: Clinical benefit of sorafenib in hepatitis $\mathrm{C}$ patients with hepatocellular carcinoma (HCC): subgroup analysis of the SHARP trial. Gastrointestinal Cancers Symposium 2008;abstr 129.
8 Craxi A, Porta C, Sangiovanni A, Seitz J, Moscovici M, Shan M, Nadel A, Voliotis D, Bruix J, Llovet JM: Efficacy and safety of sorafenib in patients with alcohol-related hepatocellular carcinoma: a sub-analysis from the SHARP trial. J Clin Oncol 2008;26(suppl):abstr 15591.

9 Bruix J, Cheng A, Kang Y, Tsao C, Qin S, Lentini G, Zou J, Nadel A, Burock K, Voliotis D, Llovet JM: Effect of macroscopic vascular invasion (MVI), extrahepatic spread (EHS), and ECOG performance status (ECOG PS) on outcome in patients with advanced hepatocellular carcinoma (HCC) treated with sorafenib: analysis of two phase III, randomized, double-blind trials. J Clin Oncol 2009;27(suppl 15):abstr 4580.

10 Ren Z, Zhu K, Kang H, Lu M, Qu Z, Lu L, Song T, Zhou W, Wang H, Yang W, Wang X, Yang Y, Shi L, Bai Y, Ye S-L: A randomized controlled phase II study of the prophylactic effect of urea-based cream on the hand-foot skin reaction associated with sorafenib in advanced hepatocellular carcinoma. ASCO Annual Meeting 2012; abstr 4008.

11 Lencioni R, Marrero J, Venook A, Ye SL, Kudo $\mathrm{M}$ : Design and rationale for the non-interventional Global Investigation of therapeutic DEcisions in hepatocellular carcinoma and Of its treatment with sorafeNib (GIDEON) study. Int J Clin Pract 2010; 64:1034-1041.

12 Faivre S, Raymond E, Boucher E, Douillard J, Lim HY, Kim JS, Zappa M, Lanzalone S, Lin X, Deprimo S, Harmon C, Ruiz-Garcia A, Lechuga MJ, Cheng AL: Safety and efficacy of sunitinib in patients with advanced hepatocellular carcinoma: an open-label, multicentre, phase II study. Lancet Oncol 2009;10:794-800.
13 Cheng A, Kang Y, Lin D, Park J, Kudo M, Qin S, Omata M, Pitman Lowenthal SW, Lanzalone S, Yang L, Lechuga M, Raymond E: Phase III trial of sunitinib (Su) versus sorafenib (So) in advanced hepatocellular carcinoma (HCC). J Clin Oncol 2011;29(suppl):abstr 4000.

14 Bhide RS, Lombardo LJ, Hunt JT, Cai ZW, Barrish JC, Galbraith S, Jeyaseelan R Sr, Mortillo S, Wautlet BS, Krishnan B, Kukral D, Malone H, Lewin AC, Henley BJ, Fargnoli J: The antiangiogenic activity in xenograft models of brivanib, a dual inhibitor of vascular endothelial growth factor receptor- 2 and fibroblast growth factor receptor-1 kinases. Mol Cancer Ther 2010;9:369-370.

15 Dailey L, Ambrosetti D, Mansukhani A, Basilico C: Mechanisms underlying differential responses to FGF signaling. Cytokine Growth Factor Rev 2005; 16:233-247.

16 Korc M, Friesel RE: The role of fibroblast growth factors in tumor growth. Curr Cancer Drug Targets 2009;9:630-651.

17 Saylor PJ, Escudier B, Michaelson MD: Importance of fibroblast growth factor receptor in neovascularization and tumor escape from antiangiogenic therapy. Clin Genitourin Cancer 2012;10:77-83.

18 Huynh H, Ngo VC, Fargnol J, Ayers M, Soo KC, Koong HN, Thng CH, Ong HS, Chung A, Chow P, Pollock P, Byron S, Tran E: Brivanib alaninate, a dual inhibitor of vascular endothelial growth factor receptor and fibroblast growth factor receptor tyrosine kinases, induces growth inhibition in mouse models of human hepatocellular carcinoma. Clin Cancer Res 2006;14:6145-6153.

19 Tovar V: FGF signaling dysregulation in HCC and role in the development of acquired resistance to anti-angiogenic therapies. HCA 2011;abstr 0-006. 
20 Finn RS, Kang YK, Mulcahy M, Polite BN, Lim HY, Walters I, Baudelet C, Manekas D, Park JW: Phase II, open-label study of brivanib as second-line therapy in patients with advanced hepatocellular carcinoma. Clin Cancer Res 2012;18:1-9.

21 Park JW, Finn RS, Kim JS, Karwal M, Li RK, Ismail F, Thomas M, Harris R, Baudelet C, Walters I, Raoul JL: Phase II, open-label study of brivanib as firstline therapy in patients with advanced hepatocellular carcinoma. Clin Cancer Res 2011;17:1973-1983.

22 Philip PA, Mahoney MR, Allmer C, et al: Phase II study of Erlotinib (OSI-774) in patients with advanced hepatocellular cancer. J Clin Oncol 2005;23:6657-6663.

23 Thomas MB, Chadha R, Glover K, Chadha R, Iwasaki M, Kaur H, Lin E, Kaseb A, Glover K, Davila M, Abbruzzese J: Phase 2 study of erlotinib in patients with unresectable hepatocellular carcinoma. Cancer 2007;110:1059-1067.

24 Thomas MB, Morris JS, Chadha R, Iwasaki M, Kaur H, Lin E, Kaseb A, Glover K, Davila M, Abbruzzese J: Phase II trial of the combination of bevacizumab and erlotinib in patients who have advanced hepatocellular carcinoma. J Clin Oncol 2009;27:843-850.

25 O’Dwyer PJ, Giantonio BJ, Levy DE, Kauh JS, Fitzgerald DB, Benson AB: Gefitinib in advanced unresectable hepatocellular carcinoma: results from the Eastern Cooperative Oncology Group's Study E1203. J Clin Oncol 2006;24(suppl):4143.

26 Ramanathan RK, Belani CP, Singh DA, Tanaka M, Lenz HJ, Yen Y, Kindler HL, Iqbal S, Longmate J, Mack PC, Lurje G, Gandour-Edwards R, Dancey J, Gandara DR: A phase II study of lapatinib in patients with advanced biliary tree and hepatocellular cancer. Cancer Chemother Pharmacol 2009;64:777-783.

27 Zhu AX, Stuart K, Blaszkowsky LS, Muzikansky A, Reitberg DP, Clark JW, Enzinger PC, Bhargava P, Meyerhardt JA, Horgan K, Fuchs CS, Ryan DP: Phase 2 study of cetuximab in patients with advanced hepatocellular carcinoma. Cancer 2007;110:581-589.

28 O'Neil BH, Goff LW, Kauh JS, Strosberg JR, Bekaii-Saab TS, Lee RM, Kazi A, Moore DT, Learoyd M, Lush RM, Sebti SM, Sullivan DM: Phase II study of the mitogen-activated protein kinase $1 / 2$ inhibitor selumetinib in patients with advanced hepatocellular carcinoma. J Clin Oncol 2011;29:2350-2356.

29 Villanueva A, Chiang DY, Newell P, et al: Pivotal role of mTOR signaling in hepatocellular carcinoma. Gastroenterology 2008;135:1972-1983, 1983.e1-11.

30 Sahin F, Kannangai R, Adegbola O, Wang J, Su G, Torbenson M: mTOR and P70 S6 kinase expression in primary liver neoplasms. Clin Cancer Res 2004;10: $8421-8425$

31 Buitrago-Molina LE, Pothiraju D, Lamle J, et al: Rapamycin delays tumor development in murine livers by inhibiting proliferation of hepatocytes with DNA damage. Hepatology 2009;50:500-509.

32 Birchmeier C, Birchmeier W, Gherardi E, Vande Woude GF: Met, metastasis, motility and more. Nat Rev Mol Cell Biol 2003;4:915-925.

33 Ueki T, Fujimoto J, Suzuki T, Yamamoto H, Okamoto E: Expression of hepatocyte growth factor and its receptor, the c-met proto-oncogene, in hepatocellular carcinoma. Hepatology 1997;25:619-623.

34 Kaposi-Novak P, Lee JS, Gomez-Quiroz L, Coulouarn C, Factor VM, Thorgeirsson SS: Met-regulated expression signature defines a subset of human hepatocellular carcinomas with poor prognosis and aggressive phenotype. J Clin Invest 2006;116:1582-1595.

35 Ke AW, Shi GM, Zhou J, Wu FZ, Ding ZB, Hu MY, Xu Y, Song ZJ, Wang ZJ, Wu JC, Bai DS, Li JC, Liu KD, Fan J: Role of overexpression of CD151 and/or c-Met in predicting prognosis of hepatocellular carcinoma. Hepatology 2009;49:491-503.

36 Cohn AL, Kelley RK, Yang T-S, Su W-C, Verslype C, Ramies DA, Lee Y, Shen X, Van Cutsem E: Activity of cabozantinib (XL184) in hepatocellular carcinoma patients (pts): results from a phase II randomized discontinuation trial (RDT). J Clin Oncol 2012;30(suppl 4):abstr 261

37 Santoro A, Rimassa L, Borbath I, et al: Tivantinib for second-line treatment of advanced hepatocellular carcinoma: a randomised, placebo-controlled phase 2 study. Lancet Oncol 2013;14:55-63.

38 Ganten T, et al: Fortschritte in der Behandlung des hepatozellulären Karzinoms. Thieme-Refresher Innere Medizin 2012;R1-R16.

39 Lipworth AD, Robert C, Zhu AX: Hand-foot syndrome (hand-foot skin reaction, palmar-plantar erythrodysesthesia): focus on sorafenib and sunitinib. Oncology 2009;77:257-271.

40 Bruix J, Raoul JL, Sherman M, Shan M, Lentini G, Nadel A, Voliotis D, Llovet JM: Efficacy and safety of sorafenib in patients with hepatocellular carcinoma (HCC): subanalysis of SHARP trial based on Barcelona Clinic Liver Cancer (BCLC) stage. J Hepatol 2009;50(suppl 1):S28-S29.

41 Finn R: Development of molecularly targeted therapies in hepatocellular carcinoma: where do we go now? Clin Cancer Res 2010;16:390-397.

42 Kelly RJ, Rixe O: Axitinib - a selective inhibitor of the vascular endothelial growth factor (VEGF) receptor. Target Oncol 2009;4:297-305.

43 Villanueva A, Llovet JM: Targeted therapies for hepatocellular carcinoma. Gastroenterology 2011;140:1410-1426. 